\title{
THE $k$ TH CONJUGATE POINT FUNCTION FOR AN EVEN ORDER LINEAR DIFFERENTIAL EQUATION
}

\author{
GEORGE W. JOHNSON
}

\begin{abstract}
For an even order, two term equation $L_{n} y=p(x) y$, $p(x)>0, x$ in $[0, \infty)$, the $k$ th conjugate point function $\eta_{k}(a)$ is defined and is shown to be a strictly increasing continuous function with domain $[0, b)$ or $[0, \infty)$. Extremal solutions are defined as nontrivial solutions with $n-1+k$ zeros on $\left[a, \eta_{k}(a)\right]$, and are shown to have exactly $n-1+k$ zeros, with even order zeros at $a$ and $\eta_{k}(a)$ and exactly $k-1$ odd order zeros in $\left(a, \eta_{k}(a)\right)$, thus establishing that $\eta_{k}(a)<\eta_{k+1}(a)$.
\end{abstract}

The differential equation considered in this paper is defined as follows: Let $p_{1}, \cdots, p_{n+1}$ be positive continuous functions defined on $[0, \infty)$, and let $A_{0}$ denote the set of all continuous functions defined on $[0, \infty)$. For $y$ in $A_{0}$, define

$$
L_{0} y=p_{1} y \text {. }
$$

Assume that $A_{i}$ and $L_{i} y$ have been defined for $i \leqq k-1$, and let $A_{k}$ denote the set of all functions $y$ for which $L_{k-1} y$ has a continuous derivative on $[0, \infty)$. For $y$ in $A_{k}$, define

$$
L_{k} y=p_{k+1}\left(L_{k-1} y\right)^{\prime} .
$$

The differential equation with which we are concerned is

$$
L_{n} y=p y,
$$

where $p$ is a positive continuous function defined on $[0, \infty)$, and $n \geqq 4$ is an even integer (cf. [3], [5], [6]).

From (1.2) and (1.3) it follows that $L_{n}$ has the factored form

$$
L_{n} y=p_{n+1}\left(p_{n}\left(\cdots p_{2}\left(p_{1} y\right)^{\prime} \cdots\right)^{\prime}\right)^{\prime} .
$$

For $y$ in $A_{n}$, the function $L_{i} y$ is said to have a zero of multiplicity $k$ at $x=a$ if

$$
L_{i} y(a)=\cdots=L_{k-1+i} y(a)=0 \quad \text { and } \quad L_{k+i} y(a) \neq 0 .
$$

Presented to the Society, April 21, 1973; received by the editors February 26, 1973. AMS (MOS) subject classifications (1970). Primary 34C10.

Key words and phrases. Extremal solution, conjugate point, zero of multiplicity $k$.

(c) American Mathematical Society 1974 
Since the functions $p_{1}, \cdots, p_{n+1}$ are positive, it follows that if $L_{k-1} y(a)=$ $L_{k-1} y(b)=0$ for some $a<b$, then there is a number $c$ in $(a, b)$ for which $L_{k} y(c)=0$. Moreover, if $L_{i} y$ has a zero of multiplicity $k$ at $x=a$, then $L_{i} y$ changes sign at $x=a$ if and only if $k$ is odd.

Denote by $N(k, a)$ the set of all points $x>a$ for which there is a nontrivial solution of (1.3), with zeros at $a$ and $x$, having at least $n-1+k$ zeros in $[a, x]$. The $k$ th conjugate point of $a$, denoted by $\eta_{k}(a)$, is defined to be the infimum of the set $N(k, a)$; if $N(k, a)$ is empty, then $\eta_{k}(a)=+\infty$. Leighton and Nehari [4] studied the functions $\eta_{k}$ extensively for the equation $\left(r y^{\prime \prime}\right)^{\prime \prime}-p y=0$ which is a special case of (1.3) with $n=4, p_{3}=r$, and $p_{i}=1$ if $i \neq 3$.

In this paper we establish the existence of $\eta_{k}(a)$ whenever $N(k, a)$ is not empty, and we consider the properties of $\eta_{k}$ as a function of $a$. We also investigate the properties of the solutions which are extremal for $\eta_{k}(a)$, in the following sense. A solution $y$ of (1.3) will be called extremal if $y$ has a zero at $a$, a zero at $\eta_{k}(a)$ and $n-1+k$ zeros in $\left[a, \eta_{k}(a)\right]$.

We can now state the main results.

THEOREM 1. If $y$ is an extremal solution for $\eta_{k}(a)$, then $y$ has even order zeros at $a$ and at $\eta_{k}(a) ; y$ has exactly $n-1+k$ zeros in $\left[a, \eta_{k}(a)\right]$, with exactly $k-1$ odd order zeros in $\left(a, \eta_{k}(a)\right) ; y$ is never zero in $(0, a)$ or in $\left(\eta_{k}(a), \infty\right)$.

THEOREM 2. As a function of $a, \eta_{k}$ is a strictly increasing continuous function whose domain is of the form $[0, b)$, or $[0, \infty)$.

In order to establish Theorem 1, we will require the following results. For notational purposes, if $y$ is a solution with $n-1+k$ zeros at $r$ points $x_{1}<\cdots<x_{r}$ we will denote by $m\left(x_{i}\right)$ the multiplicity of the zero of $y$ at $x_{i}$. Define the number

$$
M(y)=\sum_{i \in I} m\left(x_{i}\right)+\sum_{i \in . J}\left[m\left(x_{i}\right)-1\right]
$$

where

$$
I=\left\{i: m\left(x_{i}\right) \text { is even }\right\} \quad \text { and } \quad J=\left\{i: m\left(x_{i}\right) \text { is odd }\right\}
$$

LEMMA 1. If $N(m, a)$ is nonempty, then for each $k=1, \cdots, m$, there exists a kth conjugate point $\eta_{k}(a)$ and a nontrivial solution $y_{k}$ having the following properties.

(i) $a<\eta_{k}(a) \leqq \eta_{k+1}(a)$, for $k=1, \cdots, m-1$.

(ii) $y_{k}$ has at least $n-1+k$ zeros in $\left[a, \eta_{k}(a)\right]$. 
(iii) No nontrivial solution of (1.3) having a zero at a has more than $n-2+k$ zeros in $\left[a, \eta_{k}(a)\right)$.

For the proof, we observe that if $N(m, a)$ is nonempty then $N(k, a)$ is nonempty for $k \leqq m$, and if $x_{0} \in N(m, a)$ then there exist $x_{1} \leqq x_{2} \leqq \cdots \leqq$ $x_{k} \leqq x_{0}$ such that $x_{i} \in N(i, a)$. Thus $\eta_{k}(a)$ exists for each $k<m$ and $\eta_{k}(a) \leqq \eta_{k+1}(a)$, establishing part (i). Part (iii) is a direct consequence of the definition of $\eta_{k}(a)$. If $N(k, a)$ is finite, or if there exists $\varepsilon>0$ such that the intersection of $N(k, a)$ with $\left(\eta_{k}(a), \eta_{k}(a)+\varepsilon\right)$ is empty, then (ii) is immediate. Otherwise we observe that there exists a sequence $\left\{x_{i}\right\}$ converging monotonically to $\eta_{k}(a)$ and a sequence of solutions $\left\{y_{i}\right\}$ having zeros at $a$ and $x_{i}$ with at least $n-1+k$ zeros in [a, $\left.x_{i}\right]$. With no loss of generality, we may assume that $y_{i}$ has zeros at $a=t_{i_{1}}<\cdots<t_{i_{r}}=x_{i}$ and that there are integers $m_{1}, \cdots, m_{r}$ with $m_{1}+\cdots+m_{r} \geqq n-1+k$ such that $m\left(t_{i_{j}}\right)=m_{j}$ for all $j=1, \cdots, r$, and all $i$. Normalizing each solution $y_{i}$, we may apply standard compactness arguments to obtain a nontrivial solution $y$ of (1.3) and a subsequence $\left\{y_{i_{j}}\right\}$ of $\left\{y_{i}\right\}$ such that $L_{a} y_{i_{j}}$ converges uniformly to $L_{a} y$, for each $q=0, \cdots, n-1$, on $\left[a, x_{1}\right]$. Since limit points of the zeros $L_{g} y_{i}$ are zeros of $L_{g} y$ we have that $y$ must have at least $n-1+k$ zeros in $\left[a, \eta_{k}(a)\right]$ with a zero at $a$ and a zero at $\eta_{k}(a)$.

The following lemma, stated without proof, is due to Levin [5].

LEMMA 2. There does not exist a nontrivial solution of (1.3) satisfying the following boundary conditions at $x_{1}<\cdots<x_{r}$.

$$
\begin{aligned}
& L_{i} y\left(x_{j}\right)=0, i=0, \cdots, m\left(x_{j}\right)-1, j=1, \cdots, r \\
& \text { if } m\left(x_{i}\right) \text { and } m\left(x_{r}\right) \text { are odd and } \\
& m\left(x_{i}\right) \text { is even for } i=2, \cdots, r-1 .
\end{aligned}
$$

It is an immediate consequence of Lemma 2 that if $y$ is a nontrivial solution of (1.3) with $n-1+k$ zeros at the points $x_{1}<\cdots<x_{r}$ then $M(y) \leqq n$, the number of odd order zeros of $y$ must exceed $k-2$ and will equal $k-1$ only if $m\left(x_{1}\right)$ and $m\left(x_{r}\right)$ are even.

LEMMA 3. If $y$ is a nontrivial solution of (1.3) having $n-1+k$ zeros at $x_{1}<\cdots<x_{r}$ such that $M(y)<n$ then there exists a nontrivial solution of (1.3) with $n-1+k$ zeros on $\left[x_{1}, x_{r}\right)$.

There are four cases to the proof, depending on whether the zeros at $x_{1}$ and $x_{r}$ are of even or odd multiplicity. Each case is treated in a similar fashion, so we will demonstrate the case in which $m\left(x_{1}\right)$ is even and $m\left(x_{r}\right)$ is odd. Let $y_{1}, \cdots, y_{n}$ be a fundamental set of solutions of (1.3). By Lemma 
2 , the matrix

$$
Y(\varepsilon)=\left[\begin{array}{cccc}
L_{0} y_{1}\left(x_{1}\right) & L_{0} y_{2}\left(x_{1}\right) & \cdots & L_{0} y_{n}\left(x_{1}\right) \\
\cdot & \cdot & & \cdot \\
\cdot & \cdot & & \cdot \\
\cdot & \cdot & & \cdot \\
L_{a_{1}} y_{1}\left(x_{1}\right) & L_{a_{1}} y_{2}\left(x_{1}\right) & \cdots & L_{a_{1}} y_{n}\left(x_{1}\right) \\
L_{0} y_{1}\left(x_{2}\right) & L_{0} y_{2}\left(x_{2}\right) & \cdots & L_{0} y_{n}\left(x_{2}\right) \\
\cdot & \cdot & & \cdot \\
\cdot & \cdot & & \cdot \\
\cdot & \cdot & & \cdot \\
L_{0} y_{1}\left(x_{r}-\varepsilon\right) & L_{0} y_{2}\left(x_{r}-\varepsilon\right) & \cdots & L_{0} y_{n}\left(x_{r}-\varepsilon\right) \\
\cdot & \cdot & & \cdot \\
\cdot & \cdot & & \cdot \\
\cdot & \cdot & & \cdot \\
L_{a_{r}} y_{1}\left(x_{r}-\varepsilon\right) & L_{a_{r}} y_{2}\left(x_{r}-\varepsilon\right) & \cdots & L_{a_{r}} y_{n}\left(x_{r}-\varepsilon\right)
\end{array}\right]
$$

is nonsingular for all $\varepsilon>0$ sufficiently small, where $q_{1}=m\left(x_{1}\right)$; and for $i=2, \cdots, r-1, q_{i}=m\left(x_{i}\right)-1$ if $m\left(x_{i}\right)$ is even, $q_{i}=m\left(x_{i}\right)-2$ if $m\left(x_{i}\right)$ is odd and $m\left(x_{i}\right) \geqq 3$, and $q_{r}=n-M(y)-2$. Then for each $\varepsilon>0$ there is a nontrivial solution vector $c(\varepsilon)=\operatorname{col}\left(c_{1}(\varepsilon), \cdots, c_{n}(\varepsilon)\right)$ of $Y(\varepsilon) c(\varepsilon)=Y(0) c$ where $c=\operatorname{col}\left(c_{1}, \cdots, c_{n}\right)$ and $y=\sum_{i=1}^{n} c_{i} y_{i}$ is the solution with $n-1+k$ zeros in $\left[x_{1}, x_{r}\right]$. Letting $y(x, \varepsilon)=\sum_{i=1}^{n} c_{i}(\varepsilon) y_{i}(x)$, we have that as $\varepsilon \rightarrow 0$, $L_{j} y(x, \varepsilon)$ converges uniformly to $L_{j} y^{*}(x)$ for $j=0, \cdots, n-1$ where $y^{*}$ is a nontrivial solution of (1.3). Since $y-y^{*}$ satisfies the boundary conditions of Lemma 2, it must be the case that $y(x)=y^{*}(x)$ for all $x$. Now $y(x, \varepsilon)$ has an even order zero at each of the points $x_{2}, \cdots, x_{r-1}$, and hence for $\varepsilon$ sufficiently small, $y(x, \varepsilon)$ must change sign near each odd order zero of $y$. A simple count establishes that $y(x, \varepsilon)$ must have $n-1+k$ zeros in $\left[x_{1}, x_{r}-\varepsilon\right]$.

COROLlaRY 3.1. If $y$ is an extremal solution of (1.3) for $\eta_{k}(a)$, then the zeros at $a$ and $\eta_{k}(a)$ are of even multiplicity.

If either $a$ or $\eta_{k}(a)$ is of odd multiplicity, then $M(y)<n$, which contradicts the fact that $y$ is extremal.

COROLLARY 3.2. If $y$ is an extremal solution for $\eta_{k}(a)$, then $y$ has exactly $n-1+k$ zeros in $\left[a, \eta_{k}(a)\right]$.

If $y$ has $n-1+r$ zeros in $\left[a, \eta_{k}(a)\right]$, and if $r>k$, then $m\left(\eta_{k}(a)\right)>r-k$. Using the techniques of Lemma 3 with the multiplicity $m\left(\eta_{k}(a)\right)-1$ at $\eta_{k}(a)$ yields a solution with $n-2+r$ zeros in $\left[a, \eta_{k}(a)-\varepsilon\right]$. 
If an extremal solution for $\eta_{k}(a)$ has $m$ odd order zeros, then clearly $M(y)+m=n-1+k$, so that $m=k-1$. If $y$ has a zero in either $(0, a)$ or $\left(\eta_{k}(a), \infty\right)$, then $y$ satisfies the boundary conditions of Lemma 2 , yielding a contradiction. This completes the proof of Theorem 1 .

LемMА 4. If $\eta_{k}(b)<\infty$, then there exists $\delta>0$ such that for $a$ in $(b-\delta, b+\delta), \eta_{k}(a)<\infty$.

Let $y$ be an extremal solution for $\eta_{k}(b)$ with zeros at $b=x_{1}<\cdots<$ $x_{r}=\eta_{k}(b)$. Then, for each $\varepsilon$, sufficiently small, we define $y(x, \varepsilon)$ to be a solution of (1.3) having zeros of multiplicity $m\left(x_{i}\right)-1$ at $x_{i}$ if $i=1$, $r$ or if $m\left(x_{i}\right)$ is odd, a zero of multiplicity $m\left(x_{i}\right)$ at $x_{i}$ if $m\left(x_{i}\right)$ is even, $1<i<r$, and zero at $b+\varepsilon$. Then we may write

$$
y(x, \varepsilon)=\sum_{i=1}^{n} c_{i}(\varepsilon) y_{i}(x)
$$

where $\left\{y_{1}, \cdots, y_{n}\right\}$ is a fundamental set of solutions of (1.3), and with no loss of generality,

$$
\sum_{i=1}^{n} c_{i}(\varepsilon)^{2}=1
$$

Then as $\varepsilon \rightarrow 0, L_{j} y(x, \varepsilon)$ converges uniformly to $L_{j} y^{*}(x)$, where $y^{*}$ is a nontrivial solution of (1.3) satisfying

$$
y^{*}(x)=\sum_{i=1}^{n} c_{i} y_{i}(x), \quad \sum_{i=1}^{n} c_{i}^{2}=1 .
$$

If $y^{*}(x) \neq k y(x)$ for all $x$, then there is a nontrivial linear combination of $y^{*}$ and $y$ satisfying the boundary conditions in Lemma 2, which is a contradiction. Then there is a $\delta>0$ such that if $|\varepsilon|<\delta, y(x, \varepsilon)$ changes sign near each odd order zero of $y^{*}$ and near $\eta_{k}(b)$ since $y(x, \varepsilon)$ has an odd order zero at $\eta_{k}(b)$ and $y^{*}$ has an even order zero there. A simple count yields that $y(x, \varepsilon)$ has $n-1+k$ zeros in $\left[b+\varepsilon, \eta_{k}(b)+\delta\right]$, and this completes the proof, since a similar argument holds for $b-\varepsilon$.

If we define $y(x, \varepsilon)$ as in Lemma 4 at the points $x_{1}, \cdots, x_{r}$ and require that $y\left(\eta_{k}(b)-\varepsilon, \varepsilon\right)=0$ rather than $y(b-\varepsilon)=0$, for $\varepsilon>0$ then we obtain the following.

LEMMA 5. If $\eta_{k}(b)$ exists, then there is a sequence $\left\{a_{i}\right\}$ converging to $b$ in $(b-\delta, b)$ such that $\eta_{k}\left(a_{i}\right)<\eta_{k}(b)$ for each $i$.

From the preceding discussion, we have for each $\varepsilon>0$, sufficiently small, that there exists a nontrivial solution $y(x, \varepsilon)$ of $(1.3)$ with $n-1+k$ zeros in $\left[b-\varepsilon, \eta_{k}(b)\right]$. From Theorem $1, y(x, \varepsilon)$ is not an extremal solution for $\eta_{k}(b-\varepsilon)$, hence $\eta_{k}(b-\varepsilon)<\eta_{k}(b)$. 
COROllary 5.1. If $\eta_{k}(x)<\infty$ for all $x \in[a, b]$ then $\eta_{k}(a)<\eta_{k}(b)$.

Suppose to the contrary that $\eta_{k}(a) \geqq \eta_{k}(b)$. For some $\varepsilon>0, \eta_{k}(b-\varepsilon)<$ $\eta_{k}(b), b-\varepsilon>a$ and $S=\left\{x>a: \eta_{k}(x)<\eta_{k}(b-\varepsilon)\right\}$ is nonempty, and hence if $d=\inf S$, then $\eta_{k}(d)=\eta_{k}(b-\varepsilon)$. If $a<d$, then $\eta_{k}(x) \geqq \eta_{k}(b-\varepsilon) \geqq \eta_{k}(d)$ for all $x$ in $(a, d)$, contradicting Lemma 6 . Thus $a=d$. Let $x_{i}$ be a sequence in $S$ converging monotonically to $a$. Then arguments of Lemma 1 yield a sequence of extremal solutions $y_{i}$ converging to a solution $y$ of (1.3) having $n-1+k$ zeros in $\left[a, \eta_{k}(b-\varepsilon)\right]$. But this contradicts the definition of $\eta_{k}(a)$ since $\eta_{k}(a) \geqq \eta_{k}(b)>\eta_{k}(b-\varepsilon)$.

COROllary 5.2. If $\eta_{k}(b)<\infty$, then $\eta_{k}(x)<\infty$ for all $x \leqq b$.

If, to the contrary, there exists an $x<b$ for which $N(x, k)$ is empty, let $a=\sup \left\{x<b: N(x, k)\right.$ is empty\}. Then $a<b$, and $\eta_{k}(x)<\infty$ for all $x$ in $(a, b)$. From Corollary 6.1 , there is a sequence $\left\{a_{i}\right\}$ in $(a, b)$ such that $a_{i+1}<a_{i}, \eta_{k}\left(a_{i+1}\right)<\eta_{k}\left(a_{i}\right)$ and $a_{i}$ converges to $a$ as $i$ tends to $\infty$. Then the techniques of Lemma 1 yield a solution $y$ of (1.3) such that $y(a)=0$ and $y$ has $n-1+k$ zeros on $\left[a, \eta_{k}(b)\right]$, contradicting Lemma 4.

COROLlaRY 5.3. $\eta_{k}$ is continuous.

If as $x \rightarrow a-0, \eta_{k}(x) \rightarrow L<\eta_{k}(a)$, then the arguments of Lemma 1 yield a nontrivial solution of (1.3) with $n-1+k$ zeros in $\left[a, \eta_{k}(a)\right]$ since $\eta_{k}$ is increasing. If as $x \rightarrow a+0, \eta_{k}(x) \rightarrow L>\eta_{k}(a)$, let $\delta=\frac{1}{2}\left(L-\eta_{k}(a)\right)$. By Lemma 4 , there exists $\varepsilon>0$ and a solution of $y(x, \varepsilon)$ having $n-1+k$ zeros in $\left[a+\varepsilon, \eta_{k}(a)+\delta\right]$. This contradicts the fact that $\eta_{k}$ is increasing.

Corollaries 5.1, 5.2, and 5.3 complete the proof of Theorem 2 .

\section{REFERENCES}

1. J. H. Barrett, Oscillation theory of ordinary linear differential equations, Advances in Math. 3 (1969), 415-509. MR 41 \#2113.

2. E. A. Coddington and N. Levinson, Theory of ordinary differential equations, McGraw-Hill, New York, 1955. MR 16, 1022.

3. G. W. Johnson, The zeros of solutions of an even order quasi differential equation, J. Analyse Math. (to appear).

4. W. Leighton and Z. Nehari, On the oscillation of self-adjoint linear differential equations of the fourth order, Trans. Amer. Math. Soc. 89 (1958), 325-377. MR 21 \#1429.

5. A. Ju. Levin, Some problems bearing on the oscillation of solutions of linear differential equations, Dokl. Akad. Nauk SSSR 148 (1963), 512-515=Soviet Math. Dokl. 4 (1963), 121-124. MR 26 \#3972.

6. Z. Nehari, Disconjugate linear differential operators, Trans. Amer. Math. Soc. 129 (1967), 500-516. MR 36 \#2860.

Department of Mathematics, University of South Carolina, Columbia, South Carolina 29208 